\title{
A homozygous nonsense mutation in the Fukutin gene causes a Walker-Warburg syndrome phenotype
}

\author{
D Beltrán-Valero de Bernabé, H van Bokhoven, E van Beusekom, W Van den Akker, S Kant, \\ W B Dobyns, B Cormand, S Currier, B Hamel, B Talim, H Topaloglu, H G Brunner
}

J Med Genet 2003:40:845-848

$\mathrm{N}$ euronal migration is a key process in the development of the cerebral cortex. During neocortex lamination new sets of neurones proliferate at the subventricular zone and migrate alongside specialised radial glial fibres to occupy their final destinations in an "inside-out" fashion. ${ }^{1}$ More than 25 neuronal migration disorders resulting in death or improper positioning of the cortical neurones have been described in humans. ${ }^{2}$ In the cobblestone neocortex the postmitotic neurones do not respond to their stop signals, and, crossing through the neocortex, bypass the glia limitans and invade the subarachnoid space. The resulting cortex is chaotically structured, consisting of an irregular lissencephalic surface and absence of lamination.

Cobblestone lissencephalies are often seen in association with additional features, such as eye malformations and congenital muscular dystrophy. Walker-Warburg syndrome (WWS, OMIM:236670), muscle-eye-brain (MEB, OMIM: 253280), and Fukuyama congenital muscular dystrophy (FCMD, OMIM:253800) are the three major entities of this group. Patients are classified into these three entities on the

\section{Key points}

- Three rare autosomal recessive syndromes form a group of related diseases: muscle-eye-brain disease (MEB), Walker-Warburg syndrome (WWS), and Fukuyama congenital muscular dystrophy (FCMD). All share the combination of congenital muscular dystrophy and brain malformations, including a neuronal migration defect.

- The genes underlying these three disorders have been implicated in O-linked protein glycosylation: Fukutin (FCMD), POMGnT1 (MEB), and POMT1 (WWS). WWS is genetically heterogeneous, and mutations in the POMT1 gene account for approximately $20 \%$ of WWS patients.

- A candidate gene strategy was used to elucidate the genetic defect in the remaining WWS patients. A homozygous nonsense mutation in the Fukutin gene was identified in one Turkish patient. So far, Fukutin mutations have been restricted to Japanese FCMD patients, whose phenotype is clinically much less severe than WWS. The Japanese FCMD patients are predicted to maintain residual fukutin activity. In contrast, the homozygous nonsense mutation identified in the present WWS patient is predicted to cause a total loss of fukutin activity.

- This result establishes a genotype-phenotype correlation for Fukutin mutations, which may also apply to other disorders involving defective O-linked glycosylation. basis of the severity of the phenotype and the presence of syndrome specific symptoms (table 1). WWS is the most severe syndrome of the group, especially with regard to the brain phenotype. The WWS brain manifests cobblestone lissencephaly with agenesis of the corpus callosum, fusion of hemispheres, hydrocephalus, dilatation of the fourth ventricle, cerebellar hypoplasia, hydrocephalus, and sometimes

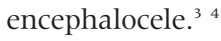

Causative genes for WWS (POMT1) $)^{5}$, FCMD (Fukutin) ${ }^{6}$ and MEB (POMGnT1 $)^{7}$ have been identified. WWS is genetically heterogeneous ${ }^{5}$, and approximately $20 \%$ of the patients show POMTI mutations. We hypothesised that severe mutations in Fukutin could give rise to a WWS phenotype.

The genotype-phenotype correlations found in FCMD patients favour this hypothesis. ${ }^{689}$ Fukutin mutations have been found only in Japanese FCMD patients. ${ }^{6}{ }^{10}$ The vast majority present at least one copy of the same mild Fukutin mutation, a $3 \mathrm{~kb}$ insertion in the $3^{\prime}$ UTR. Patients homozygous for this insertion show a milder phenotype than do compound heterozygotes, carrying the insertion in combination with a missense or nonsense mutation on the other allele (fig 1B). We therefore postulated that homozygous nonsense mutations would give rise to a more severe phenotype. This hypothesis is supported by the fact that Fukutin deficient chimeric mice show a severe phenotype which closely resembles WWS. ${ }^{1}$

Based on this premise, we did a linkage study in 30 consanguineous WWS families. These patients have been diagnosed as WWS according to Cormand et al. ${ }^{3}$ In brief, patients with WWS die before the age of three years and have a thin cerebral mantle, a smooth cerebral surface, severe cerebellar/vermis hypoplasia, absent corpus callosum, and fusion of hemispheres. Two markers flanking the FCMD locus were selected: D9S1784, located $0.30 \mathrm{Mb}$ centromeric to FCMD, and D9S172, located $0.27 \mathrm{Mb}$ telomeric. Homozygosity was observed in three of the 30 patients, each of them with different haplotypes: patient 1, D9S1784:180/ 180, D9S172:299/299; patient 2, D9S1784:186/186, D9S172:303/303; and patient 3, D9S1784:192/192, D9S172: 303/303.

Amplification of each of the 10 exons and flanking intron sequences of the FCMD gene and mutation analysis was carried out by direct sequencing. ${ }^{8}$ A homozygous mutation was found in one of the three patients linked to the FCMD locus. Patient $\mathrm{l}$ is homozygous for a novel dinucleotide substitution c.345_346GC $\rightarrow$ CT that creates a Glnll6Stop mutation in exon 4 (fig 1 ). The mendelian segregation of the mutation Gln116Stop was confirmed by direct sequencing of family members. The Glnl16Stop mutation was not

Abbreviations: FCMD, Fukuyama congenital muscular dystrophy; MEB, muscle-eye-brain disease; WWS, Walker-Warburg syndrome 
A

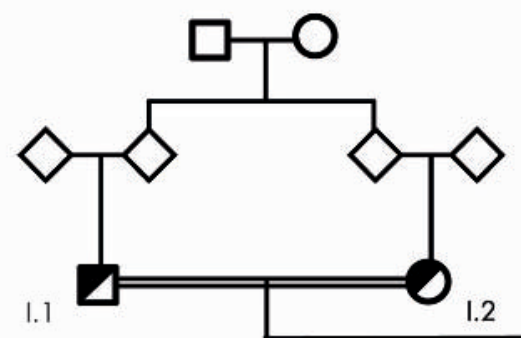

II. 1

II. 2

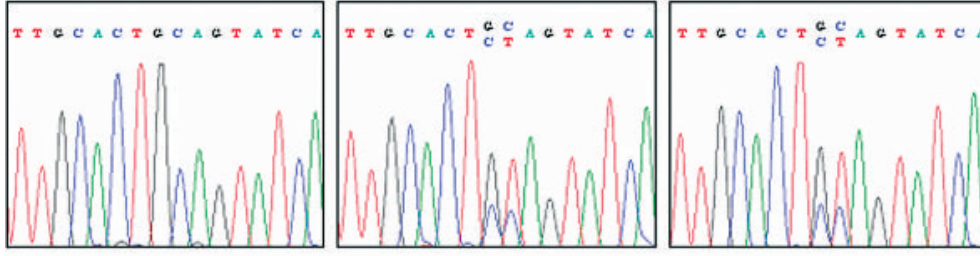

Control
I. 1
1.2

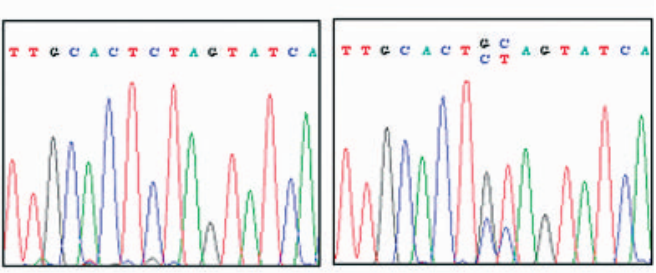

II. 1
$\| .2$

B

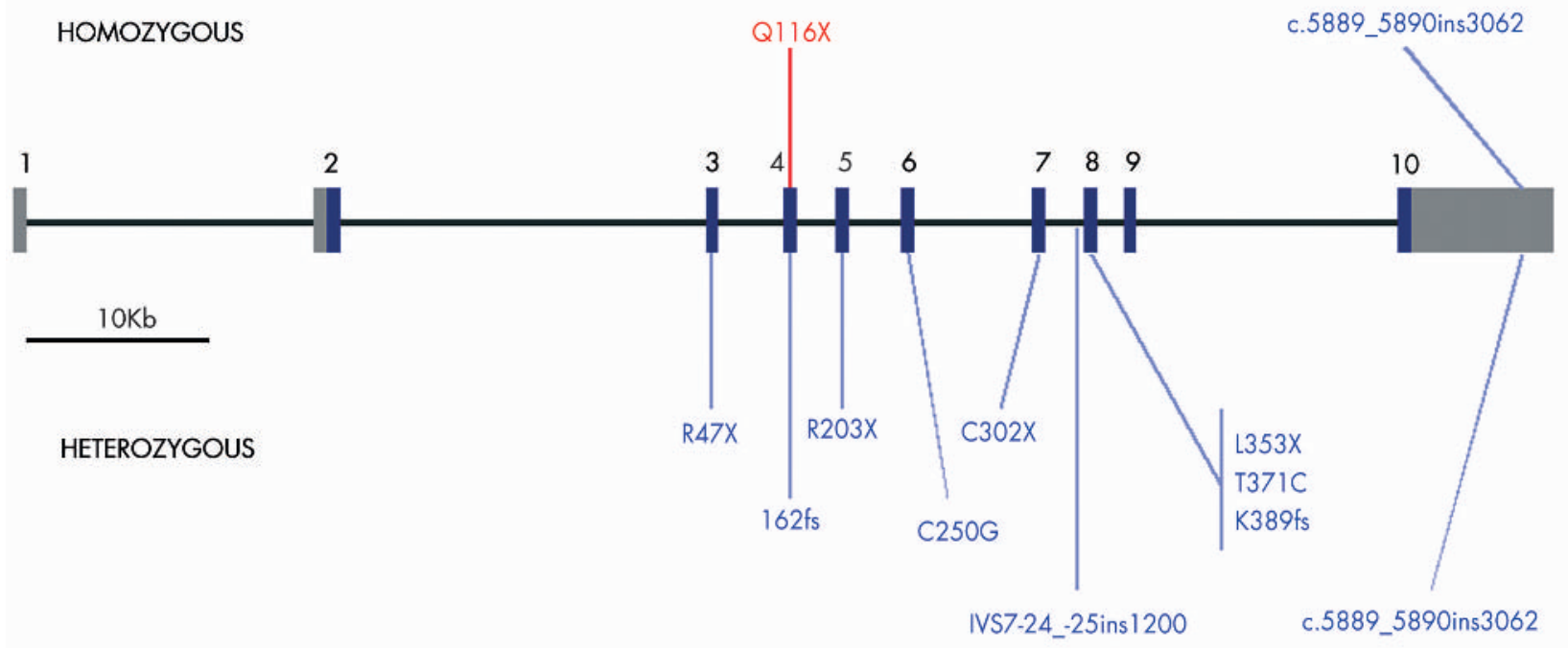

Figure 1 Mutations in the Fukutin gene. (A) The pedigree and the chromatograms corresponding to family 1. (B) The mutations described to date in Fukutin. Mutations found in FCMD patients are in blue, while the mutation found in WWS family 1 is in red. The mutations shown below the schematic representation of the Fukutin gene structure have been found in heterozygosis, and the mutations on top in homozygosis. FCMD patients are compound heterozygous or homozygous for the mutation c.5889_5890ins3062. 
Table 1 Clinical features of patient 1 compared with cobblestone lissencephalies

\begin{tabular}{|c|c|c|c|c|}
\hline Symptom ${ }^{1}$ & MEB & WWS & Patient 1 & FCMD \\
\hline Cortical abnormality & $\begin{array}{l}\text { Pachygyria/ } \\
\text { polymicrogyria }\end{array}$ & $\begin{array}{l}\text { Agyria thin } \\
\text { mantle }\end{array}$ & $\begin{array}{l}\text { Agyria/ } \\
\text { pachygyria }\end{array}$ & $\begin{array}{l}\text { Pachygyria/ } \\
\text { polymicrogyria }\end{array}$ \\
\hline Encephalocele & - & + & - & \\
\hline Dilated ventricles & ++ & ++ & +++ & + \\
\hline White matter abnormality & + & +++ & +++ & + \\
\hline Fused hemispheres & - & + & ++ & + \\
\hline Absent septum & - & + & + & - \\
\hline Corpus callosum & Normal/thin & Absent & Absent & Normal/thin \\
\hline Cerebellar hypoplasia & ++ & ++ & +++ & - \\
\hline Cerebellar vermis & Hypoplastic & Absent & Absent & Normal \\
\hline Muscular dystrophy & +++ & ++ & +++ & ++ \\
\hline Retinal defects & ++ & ++ & + & - \\
\hline Anterior chamber abnormalities & ++ & ++ & +++ & + \\
\hline Life expectancy & $10-30$ years & $<3$ years & $4 \frac{1}{2}$ months & $10-20$ years \\
\hline Distribution & Mostly Finland & Worldwide & Turkey & Japan \\
\hline \multicolumn{5}{|c|}{$\begin{array}{l}\text { 'The severity of the symptoms is graded from - (absent) to }+++ \text { (very pronounced). } \\
\text { FCMD, Fukuyama congenital muscular dystrophy; MEB, muscle-eye-brain disease; WWS, Walker-Warburg } \\
\text { syndrome. }\end{array}$} \\
\hline
\end{tabular}
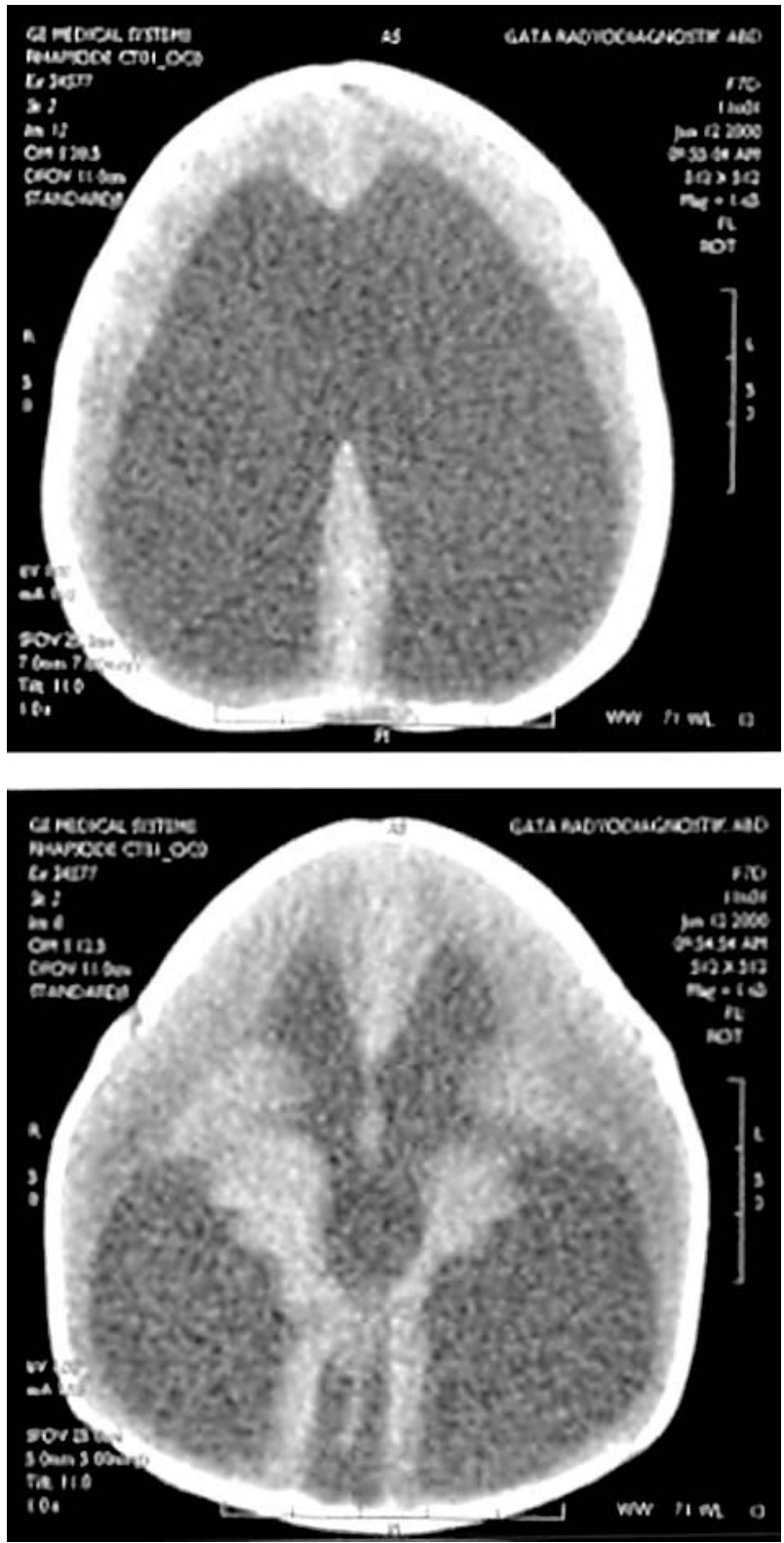

Figure 2 Cranial computed tomography on day 18 showing severe hydrocephalus with very marked pachygyria, almost to the extent of agyria, white matter hyperlucency, absent corpus callosum, and cerebellar hypoplasia. found in an SSCP based screening of 60 Dutch and 45 Turkish controls, indicating that this mutation is causative for the WWS phenotype. Patient 2 is homozygous for a transition c.1336A $\rightarrow \mathrm{G}$ in exon 10, predicting a Asn446Asp substitution. Asn446 is conserved in Fukutin orthologues from different species, but is unlikely to be a causative mutation, as it was found with a frequency of 3\% in the normal population (data not shown). No mutation was detected in patient 3 .

Patient 1 is a Turkish boy born to second degree consanguineous parents at term with a birth weight of 2945 g. At birth he had macrocephaly, abnormal eyes, and severe hypotonia (fig 2; table 1). Delivery was complicated by asphyxia and meconium aspiration. His 5 and 10 minute Apgar scores were 2 and 4, respectively. When examined at the age of four weeks, he was hypotonic, and spontaneous movements were diminished. He could not maintain head control on ventral suspension. Sucking was weak, but grasp and rooting reflexes were present. Bilateral corneal clouding and megalocornea were noted. Fundus examination showed severe myopic changes. Rapid growth in head circumference was noticed during the neonatal period. Cranial computed tomography at 18 days showed severe hydrocephalus, brain stem hypoplasia, and abnormal gyral patterns, along with white matter hyperlucency and absence of the corpus callosum. Serum creatine kinase on days 15 and 18 was raised at $1842 \mathrm{U} / \mathrm{l}$ and $6983 \mathrm{U} / \mathrm{l}$, respectively. A muscle biopsy taken from the quadriceps contained only a few muscle cells and was not suitable for interpretation. The patient was diagnosed as having Walker-Warburg syndrome. He died at $4 \frac{1}{2}$ months of age.

The mutation described here is the first Fukutin mutation found in a non-Japanese patient. The propositus represents the most severe end of the phenotypic spectrum of Fukutin mutations. FCMD patients homozygous for the founder Japanese mutation show a relatively mild phenotype which clearly differs from WWS, while the compound heterozygotes show more severe symptoms such as microphthalmia and pronounced hydrocephalus. ${ }^{8}{ }^{10}$ The present patient clearly has an even more severe phenotype which matches the established criteria for a WWS phenotype (table 1). ${ }^{3}$

The broad phenotypic spectrum associated with Fukutin mutations suggests that other intermediate cobblestone phenotypes, such as MEB, may also be caused by mutations in the Fukutin gene. An analogous situation is seen for mutations in the fukutin related protein gene (FKRP). Mutations in FKRP give rise to a broad clinical spectrum 
that ranges from mild muscular dystrophy (LGMD2I) to the more severe form (MDCIC), which in some cases is also associated with intellectual impairment and cerebellar cysts. ${ }^{12}$ The severity of the observed phenotypes is a reflection of the severity of the FKRP mutations found in these patients. We envisage that the clinical heterogeneity seen for mild and severe mutations of Fukutin and FKRP may also apply to POMGnTl and POMT1. We hypothesise that milder mutations in POMT1 may cause a phenotype resembling FCMD or MEB rather than WWS. Similarly, complete loss of function has not been demonstrated for POMGnTl mutations in MEBD. ${ }^{7}$ Therefore, more severe mutations in POMGnTl, if these exist, may be associated with a WWS phenotype. However, as in the case of Fukutin, mutations in POMGnTl and FKRP may explain only a small fraction of the large number of unsolved WWS cases.

\section{NOTE ADDED IN PROOF}

While this paper was being written, a similar case was described by Silan et al (Ann Neurol 53:392-6). In that article a Turkish patient with a WWS phenotype is described, presenting a homozygous nonsense mutation in Fukutin exon 5. This observation is consistent with the notion of a genotype-phenotype correlation for Fukutin mutations.

\section{ACKNOWLEDGEMENTS}

This work was supported by grants from the Dutch Foundation for Scientic Research (NWO, 903-42-190) and the Prinses Beatrix Fonds en de Stichting Spieren voor Spieren (MAR00-117; MAR02-0226).

\section{ELECTRONIC DATABASE INFORMATION}

Accession numbers and URLs for data presented herein are as follows:

GenBank, http://www.ncbi.nlm.nih.gov/Genbank/ (for Fukutin [accession number NM 006731]).

Online Mendelian Inheritance in Man (OMIM), http:// www.ncbi.nlm.nih.gov/Omim/ (for WWS [MIM 236670], MEB [MIM 253280], FCMD [MIM 253800], LGMD2I [607155]).

\section{Authors' affiliations}

D Beltrán-Valero de Bernabé, $\mathrm{H}$ van Bokhoven, $\mathrm{E}$ van Beusekom, B Hamel, H G Brunner, Department of Human Genetics, University Medical Centre Nijmegen, Nijmegen, Netherlands
W Van den Akker, Netherlands Institute for Developmental Biology/ Hubrecht Laboratory, Utrecht, Netherlands

S Kant, Department of Clinical Genetics, University Medical Centre Leiden, Leiden, Netherlands

W B Dobyns, Departments of Human Genetics, Neurology, and Pediatrics, The University of Chicago, Chicago, Illinois, USA

B Cormand, Department of Genetics, University of Barcelona, Barcelona, Spain

S Currier, Division of Neurogenetics, Beth Israel Deaconess Medical Center and Harvard Medical School, Boston, Massachusetts, USA

B Talim, H Topaloglu, Hacettepe Children's Hospital, Ankara, Turkey

Correspondence to: Dr Han G Brunner, 417 Department of Human Genetics, PO Box 9101, 6500 HB Nijmegen, Netherlands; h.brunner@antrg.umcn.nl

\section{REFERENCES}

1 Marin-Padilla M. Dual origin of the mammalian neocortex and evolution of the cortical plate. Anat Embryol (Berl) 1978;152:109-26.

2 Lammens M. Neuronal migration disorders in man. Eur $J$ Morphol 2000;38:327-33.

3 Cormand B, Pihko $\mathrm{H}$, Bayes $\mathrm{M}$, et al. Clinical and genetic distinction between Walker-Warburg syndrome and muscle-eye-brain disease. Neurology 2001;56:1059-69

4 Dobyns WB, Pagon RA, Armstrong D, et al. Diagnostic criteria for WalkerWarburg syndrome. Am J Med Genet 1989:32:195-210.

5 Beltrán-Valero de Bernabé D, Currier S, Steinbrecher A, et al. Mutations in the O-mannosyltransferase gene POMT1 give rise to the severe neuronal migration disorder Walker-Warburg syndrome. Am J Hum Genet 2002;71:1033-43

6 Kobayashi K, Nakahori Y, Miyake M, et al. An ancient retrotransposal insertion causes Fukuyama-type congenital muscular dystrophy. Nature 1998;394:388-92

7 Yoshida A, Kobayashi K, Manya H, et al. Muscular dystrophy and neuronal migration disorder caused by mutations in a glycosyltransferase, POMGnT1. Dev Cell 2001:1:717-24.

8 Kondo-Lida E, Kobayashi K, Watanabe M, et al. Novel mutations and genotype-phenotype relationships in 107 families with Fukuyama-type congenital muscular dystrophy (FCMD). Hum Mol Genet 1999:8:2303-9.

9 Saito K, Osawa M, Wang ZP, et al. Haplotype-phenotype correlation in Fukuyama congenital muscular dystrophy. Am J Med Genet 2000;92: 184-90.

10 Kobayashi K, Sasaki J, Kondo-lida E, et al. Structural organization, complete genomic sequences and mutational analyses of the Fukuyama-type congenital muscular dystrophy gene, fukutin. FEBS Lett 2001;489:192-6

11 Takeda S, Kondo M, Sasaki J, et al. Fukutin is required for maintenance of muscle integrity, cortical histogenesis, and normal eye development. Poster 2199 presented at the 53rd Annual ASHG Meeting. Am J Hum Genet 2002;71:545.

12 Topaloglu $H$, Brockington $M$, Yuva Y, et al. FKRP gene mutations cause congenital muscular dystrophy, mental retardation and cerebellar cysts. Neurology 2003;60:988-92. 